\title{
MENINGITIS NEONATAL EN UN HOSPITAL GENERAL DE LIMA, PERÚ, 2008 AL 2015
}

\author{
George Lewis ${ }^{1, a}$, Maggie Schweig ${ }^{1, a}$, Daniel Guillén-Pinto, ${ }^{1,2, b}$, María Luz Rospigliosi, 1,2,c
}

\begin{abstract}
RESUMEN
Con el objetivo de describir la incidencia, características clínicas y factores asociados a la meningitis neonatal (MN) se realizó un estudio de serie de casos, del 2008 al 2015, donde se incluyeron 53 neonatos, 10 con MN de inicio temprano y 43 de inicio tardío. Se encontró una incidencia de 1,5 por mil nacidos vivos y una letalidad de 3,8\%. Los factores asociados fueron fiebre periparto, infección urinaria y corioamnionitis $(p<0,05)$. Los síntomas frecuentes fueron fiebre $(84,9 \%)$, hipotonía $(77,4 \%)$ e hipoactividad $(73,6 \%)$. En el líquido cefalorraquídeo (LCR), los casos de MN temprana presentaron una mediana de 330 leucocitos $/ \mu \mathrm{L}$ y $29 \mathrm{mg} / \mathrm{dL}$ de glucosa, y en los casos de $\mathrm{MN}$ tardía presentaron 170 leucocitos/ $\mu \mathrm{L}$ y $32 \mathrm{mg} / \mathrm{dL}$ de glucosa. Las bacterias más frecuentes fueron Listeria monocytogenes (16,9\%) y Staphylococcus coagulasa negativo (11,3\%). En conclusión, la incidencia fue alta en comparación con otros estudios y los factores asociados fueron los esperados.
\end{abstract}

Palabras clave: Meningitis; Recién nacido, Prematuro, Sepsis, Listeria monocytogenes (fuente: DECS BIREME).

\section{NEONATAL MENINGITIS IN A GENERAL HOSPITAL IN LIMA, PERU, 2008 TO 2015}

\begin{abstract}
Aiming at describing the incidence, clinical characteristics and factors associated with neonatal meningitis (NM), a case series study was conducted from 2008 to 2015, where 53 neonates, 10 with early onset NM and 43 with late onset were included. An incidence of 1.5 per thousand live births and a lethality of $3.8 \%$ were found. The associated factors were peripartum fever, urinary tract infection and chorioamnionitis $(P<0.05)$. Frequent symptoms were fever $(84.9 \%)$, hypotonia $(77.4 \%)$ and hypoactivity $(73.6 \%)$. In the cerebrospinal fluid (CSF), the cases of early NM presented a median of 330 leukocytes $/ \mu \mathrm{l}$ and $29 \mathrm{mg} / \mathrm{dl}$ of glucose, and the cases of late NM presented 170 leukocytes/ $\mu \mathrm{l}$ and $32 \mathrm{mg} / \mathrm{dl}$ of glucose. The most common bacteria were Listeria monocytogenes $(16.9 \%)$ and negative coagulase staphylococcus (11.3\%). In conclusion, the incidence was high compared to other studies and the associated factors were the expected.
\end{abstract}

Key words: Meningitis; Infant, newborn; Infant, premature; Sepsis, Listeria monocytogenes (Source: MeSH NLM).

\section{INTRODUCCIÓN}

La meningitis neonatal (MN) es una enfermedad grave con gran impacto sobre la morbimortalidad infantil. La incidencia mundial oscila entre 0,8 y 6,1 casos por mil nacidos vivos $y$, en el Perú, dos series la describen entre 0,5 y 0,9 casos por mil nacidos vivos ${ }^{(1-4)}$. La letalidad es elevada: $10 \%$ en países desarrollados y $40-58 \%$ en países en desarrollo ${ }^{(3)}$. Con frecuencia los sobrevivientes presentan secuelas neurológicas (20-60\%), ello incluye retraso mental y del lenguaje, epilepsia y parálisis cerebral ${ }^{(1,5)}$. El bajo peso y la prematuridad se asocian con un peor pronóstico ${ }^{(3)}$.

Se describen dos formas clínicas: de aparición temprana, cuando la enfermedad se inicia en los primeros 3 días de vida, ocasionado generalmente por microorganismos del canal del parto, y de aparición tardía, cuando se inicia después de los tres días, habitualmente por patógenos intrahospitalarios. Sin embargo, algunos autores consideran puntos de corte variables. ${ }^{(1,4,6,7)}$.

Los factores de riesgo descritos para la MN temprana son corioamnionitis, infecciones del tracto urinario (ITU), infecciones vaginales, ruptura prematura de membranas (RPM) y fiebre intraparto. En cambio, para la tardía se asocian factores relacionados con terapia intensiva como procedimientos invasivos y técnicas neuroquirúrgicas ${ }^{(2,3,8,9)}$. La frecuencia de aislamiento del patógeno es variable, $14 \%$ hasta $78 \%$, y usualmente se describen Streptococcus del grupo B (GBS), Escherichia coli y Listeria monocytogenes ${ }^{(2,3,10-12)}$.

\footnotetext{
Universidad Peruana Cayetano Heredia, Lima, Perú

Hospital Cayetano Heredia, Lima, Perú

Médico cirujano; ${ }^{\mathrm{b}}$ neurólogo pediatra; ${ }^{\mathrm{c}}$ pediatra neonatóloga

Recibido: 27/07/2016 Aprobado: 22/02/2017 En línea: 28/06/2017
}

Citar como: Lewis G, Schweig M, Guillén-Pinto D, Rospigliosi ML. Meningitis neonatal en un hospital general de Lima, Perú, 2008 al 2015. Rev Peru Med Exp Salud Publica. 2017;34(2):233-8. doi: 10.17843/rpmesp.2017.342.2297 
El objetivo de este estudio fue determinar la incidencia hospitalaria y describir las características demográficas, clínicas y laboratoriales de los casos de MN atendidos en un hospital general.

\section{EL ESTUDIO}

Se realizó un estudio descriptivo, tipo serie de casos, en el Servicio de Neuropediatría del Hospital Cayetano Heredia $(\mathrm{HCH})$, de enero de 2008 a diciembre de 2015. El HCH es el hospital de mayor nivel de referencia de la zona norte de Lima, con una población de procedencia urbano marginal.

Se incluyeron los casos confirmados y probables de MN atendidos en este periodo. Se excluyeron aquellos sin historia clínica, sin registro de los datos para establecer el diagnóstico o con malformaciones neurológicas severas.

Los casos confirmados fueron neonatos a término $<28$ días, o pretérmino $<44$ semanas de edad corregida, con pleocitosis en liquido cefalorraquídeo $(\mathrm{LCR}) \geq 30$ células $/ \mu \mathrm{L}$ e identificación del agente. Los casos probables fueron aquellos con pleocitosis $\geq 30$ células/ $\mu \mathrm{L}$ sin confirmación del patógeno. Se tomó como punto de corte entre $\mathrm{MN}$ temprana y tardía a los tres días de vida al ser este el estándar considerado en nuestra institución.

Las variables consideradas para el estudio fueron: el tipo de parto, edad gestacional, peso al nacer, factores maternos y neonatales asociados, procedimientos realizados, sintomatología descrita en $\mathrm{HC}$, edad al diagnóstico (en días), pleocitosis en LCR, porcentaje de polimorfonucleares en LCR, glucosa en LCR, hematíes en LCR y el patógeno aislado.

Los datos obtenidos fueron analizados mediante frecuencias y porcentajes y las pruebas de Fisher y $U$

\section{MENSAJES CLAVE}

Motivación para realizar el estudio. La meningitis neonatal (MN) es una enfermedad con alto riesgo de mortalidad y secuelas severas en los supervivientes, por lo cual su diagnóstico oportuno es esencial; sin embargo, la información disponible acerca de esta enfermedad en el Perú es escasa.

Principales hallazgos. Los síntomas identificados en esta enfermedad son poco específicos (fiebre, hipotonía e irritabilidad) por lo cual se requiere una alta sospecha para el diagnóstico. La bacteria identificada con mayor frecuencia en nuestro estudio fue Listeria monocytogenes y estafilococo coagulasa negativo.

Implicancias. La bacteriología de la MN en esta cohorte peruana difiere de lo ya descrito. Nuestros hallazgos deben ser corroborados en estudios posteriores

de Mann-Whitney con nivel de significancia del 5\%, empleando el programa Minitab versión 15. Se obtuvo la autorización de los comités de ética de la Universidad Peruana Cayetano Heredia (UPCH) y del HCH. Los datos fueron codificados para su confidencialidad.

\section{RESULTADOS}

Se encontraron 62 casos con diagnóstico de MN del 2008 al 2015. De estos, se excluyeron nueve por falta de la historia clínica. Ninguno presentó malformaciones severas. De los 53 seleccionados, $39 \%$ fueron pretérmino, $58,5 \%$ de sexo femenino y $3,8 \%$ fallecieron.

La incidencia estimada fue de 1,5 por mil nacidos vivos, determinada a partir de los 36512 nacimientos en el periodo de estudio. Esta fue mayor en neonatos de muy bajo peso y en < 34 semanas; 17,2 y 15,5 casos por mil nacidos vivos, respectivamente (Tabla 1 ).

Tabla 1. Características clínicas de la población de estudio con meningitis neonatal en HCH, 2008-2015

\begin{tabular}{|c|c|c|c|}
\hline Características clínicas $n(\%)$ & $\begin{array}{l}\text { MN de aparición temprana } \\
(\mathrm{n}=10)\end{array}$ & $\begin{array}{l}\text { MN de aparición tardía } \\
(n=43)\end{array}$ & Valor $\mathrm{p}^{*}$ \\
\hline Tipo de parto & & & 0,29 \\
\hline Vaginal & $7(70,0)$ & $20(46,5)$ & \\
\hline Cesárea & $3(30,0)$ & $23(53,5)$ & \\
\hline Sexo & & & 0,49 \\
\hline Masculino & $3(30,0)$ & $19(44,2)$ & \\
\hline Femenino & $7(70,0)$ & $24(55,8)$ & \\
\hline Edad gestacional & & & 0,49 \\
\hline$<34$ semanas & $2(20,0)$ & $9(21,0)$ & \\
\hline 34 a 36 semanas & $3(30,0)$ & $7(16,3)$ & \\
\hline$\geq 37$ semanas & $5(50,0)$ & $27(62,7)$ & \\
\hline Peso al nacer & & & 0,24 \\
\hline$<1500$ gramos & $1(10,0)$ & $8(18,7)$ & \\
\hline $1500-2499$ gramos & $4(40,0)$ & $6(13,9)$ & \\
\hline$\geq 2500$ gramos & $5(50,0)$ & $29(67,4)$ & \\
\hline Fallecidos & $1(10,0)$ & $1(2,3)$ & 0,344 \\
\hline Patógeno aislado & $6(60,0)$ & $16(37,2)$ & 0,29 \\
\hline
\end{tabular}

*Prueba exacta de Fisher

MN: meningitis neonatal, $\mathrm{HCH}$ : Hospital Cayetano Heredia 
De los casos, diez fueron de aparición temprana y 43 de aparición tardía. La mediana de la edad al diagnóstico fue un día en casos tempranos y de 11 en casos tardíos. El agente causal se aisló en 22 casos (41,5\%), seis $(60,0 \%)$ en casos tempranos y $16(37,2 \%)$ en casos tardíos, cumpliendo así con la definición de casos confirmados. Fallecieron dos casos, uno temprano y uno tardío (Tabla 1).

Los factores maternos más frecuentes en la $\mathrm{MN}$ de aparición temprana fueron: fiebre periparto, ITU y corioamnionitis, mientras que en la tardía fueron: fiebre periparto y corioamnionitis. Se puede apreciar que la corioamnionitis, la fiebre periparto y la ITU son los únicos factores que demostraron asociación significante ( $p=0,035, p=0,008$ y $p<0,001$, respectivamente).

Los factores neonatales predominantes en la $\mathrm{MN}$ temprana fueron neumonía connatal, ictericia patológica, hipoglicemia y sepsis, mientras que en la MN tardía fueron ictericia patológica y sepsis. La fiebre, hipotonía e hipoactividad, se encontraron en más del $70 \%$ de los casos. No hubo diferencias estadísticamente significativas en la sintomatología según la forma de presentación (Tabla 2).

Tabla 2. Factores asociados y síntomas en la población de estudio con meningitis neonatal en HCH, 2008-2015

\begin{tabular}{|c|c|c|c|}
\hline Características n(\%) & $\begin{array}{l}\text { MN de aparición temprana } \\
(\mathrm{n}=10)\end{array}$ & $\begin{array}{c}\text { MN de aparición tardía } \\
(n=43)\end{array}$ & Valor $\mathrm{p}^{*}$ \\
\hline \multicolumn{4}{|l|}{ Maternos } \\
\hline Corioamnionitis & $5(50,0)$ & $7(16,3)$ & 0,035 \\
\hline Fiebre periparto & $7(70,0)$ & $10(23,3)$ & 0,008 \\
\hline Ruptura prematura de membranas $>18 \mathrm{~h}$ & $0(0,0)$ & $5(11,6)$ & 1 \\
\hline Infección vaginal & $1(10,0)$ & $4(9,3)$ & 1 \\
\hline Infección del tracto urinario & $7(70)$ & $5(11,6)$ & $<0,001$ \\
\hline Líquido amniótico meconial & $3(30,0)$ & $3(6,9)$ & 0,073 \\
\hline$\geq 1$ factores de riesgo & $10(100,0)$ & $17(39,5)$ & $<0,001$ \\
\hline \multicolumn{4}{|l|}{ Neonatales } \\
\hline Sepsis & $3(30,0)$ & $19(44,2)$ & 0,49 \\
\hline Encefalopatía hipóxico-isquémica & $0(0,0)$ & $1(2,3)$ & 1 \\
\hline Hemorragia intraventricular & $1(10,0)$ & $8(18,6)$ & 1 \\
\hline Síndrome de distrés respiratorio & $1(10,0)$ & $10(23,3)$ & 0,67 \\
\hline Neumonía connatal & $4(40,0)$ & $6(13,9)$ & 0,08 \\
\hline Infección del tracto urinario & $1(10,0)$ & $6(13,9)$ & 1 \\
\hline Gastroenterocolitis aguda & $1(10,0)$ & $4(9,3)$ & 1 \\
\hline Cardiopatía congénita & $0(0,0)$ & $11(25,6)$ & 0,09 \\
\hline Ictericia patológica & $3(30)$ & $21(48,8)$ & 0,32 \\
\hline Anemia & $1(10,0)$ & $7(16,3)$ & 1 \\
\hline Hipoglicemia & $3(30,0)$ & $5(11,6)$ & 0,16 \\
\hline Enfermedad renal & $2(20,0)$ & $2(4,6)$ & 0,15 \\
\hline Alteración hidroelectrolítica & $0(0,0)$ & $4(9,3)$ & 1 \\
\hline$\geq 1$ factores de riesgo & $9(90,0)$ & $38(88,4)$ & 1 \\
\hline \multicolumn{4}{|l|}{ Procedimientos $\mathrm{n}(\%)$} \\
\hline Cateterismo arterial & $1(10,0)$ & $9(20,9)$ & 0,67 \\
\hline Cateterismo venoso periférico & $2(20,0)$ & $8(18,6)$ & 1 \\
\hline Catéter venoso central & $1(10,0)$ & $5(11,6)$ & 1 \\
\hline Catéter central de inserción periférica & $5(50,0)$ & $13(30,2)$ & 0,28 \\
\hline Nutrición parenteral total & $2(20,0)$ & $12(27,9)$ & 1 \\
\hline Ventilación mecánica & $6(60,0)$ & $16(37,2)$ & 0,28 \\
\hline Cirugía & $0(0,0)$ & $3(6,9)$ & 1 \\
\hline$\geq 1$ factores de riesgo & $7(70,0)$ & $28(65,1)$ & 1 \\
\hline \multicolumn{4}{|l|}{ Síntomas } \\
\hline Fiebre & $7(70,0)$ & $38(88,4)$ & 0,60 \\
\hline Hipotonía & $9(90,0)$ & $32(74,4)$ & 0,42 \\
\hline Hipoactividad & $8(80,0)$ & $31(72,1)$ & 1 \\
\hline Ictericia & $5(50,0)$ & $25(58,1)$ & 0,73 \\
\hline Irritabilidad & $4(40,0)$ & $22(51,2)$ & 0,73 \\
\hline Dificultad respiratoria & $5(50,0)$ & $16(37,2)$ & 0,49 \\
\hline Hiporreflexia & $4(40,0)$ & $16(37,2)$ & 1 \\
\hline Hiperreflexia & $3(30,0)$ & $12(27,9)$ & 1 \\
\hline Abombamiento de fontanelas & $2(20,0)$ & $7(16,3)$ & 1 \\
\hline Apnea & $1(10,0)$ & $8(18,6)$ & 1 \\
\hline Síntomas respiratorios altos & $0(0,0)$ & $8(18,6)$ & 0,33 \\
\hline Hipertonía & $1(10,0)$ & $7(16,3)$ & 1 \\
\hline Crisis convulsiva & $1(10,0)$ & $7(16,3)$ & 1 \\
\hline
\end{tabular}

*Prueba exacta de Fisher

MN: meningitis neonatal, $\mathrm{HCH}$ : Hospital Cayetano Heredia 
Para la MN temprana, la mediana del tiempo de enfermedad, previo a la punción lumbar (PL), fue 1,5 días (0-3 días) mientras que para la tardía fue dos días (1-12 días). La mediana del número de PL realizadas fue de dos en ambas formas. En la MN temprana la mediana de leucocitos fue $330 / \mu \mathrm{L}$, glucosa $29 \mathrm{mg} / \mathrm{dL}$ y proteínas $228,5 \mathrm{mg} / \mathrm{dL}$, mientras que en la tardía fue 170 leucocitos/ $\mu \mathrm{L}, 32 \mathrm{mg} / \mathrm{dL}$ de glucosa y $190 \mathrm{mg} / \mathrm{dL}$ de proteínas (Tabla 3). Los patógenos más frecuentes fueron Listeria monocytogenes (2/10 en MN temprana y 7/43 en MN tardía) y Staphylococcus coagulasa negativo (1/10 en MN temprana y 5/43 en MN tardía). En tres casos se obtuvo el microorganismo tanto en sangre como en LCR. (Tabla 4).

\section{DISCUSIÓN}

La MN es una enfermedad poco diagnosticada y subregistrada en nuestro medio. Hecho inconsistente por la gran frecuencia casos de sepsis neonatal reportada entre las primeras causas de morbimortalidad infantil en el Perú (12).

La incidencia en este estudio fue superior a la reportada en el 2008 en el mismo hospital (0,9 casos por mil nacidos vivos) y en el año 1993 en el Hospital Rebagliati $\left(0,47\right.$ casos por mil nacidos vivos) ${ }^{(1,2)}$. El aumento se relacionaría con una mejora en el diagnóstico, mayor complejidad asistencial y mejor capacidad resolutiva. Sin embargo, se encuentra dentro del rango esperado a nivel internacional ${ }^{(3,10,13,14)}$.

La forma tardía fue notoriamente más frecuente que la temprana (4,3 veces), esto es semejante a lo descrito en una revisión sistemática, que demostró mayor riesgo de infección grave en neonatos hospitalizados por tiempo prolongado ${ }^{(5)}$. Este patrón puede variar entre diferentes unidades neonatales ${ }^{(10)}$ y en una misma, al cambiar las condiciones asistenciales ${ }^{(1)}$.

Aún persiste controversia con respecto al punto de corte para definir MN temprana y tardía; algunos refieren el corte en 3 días ${ }^{(2,4)}$, otros en 7 días ${ }^{(1,6,9)}$ y otros consideran puntos variables ${ }^{(5)}$. En este estudio, la mediana de edad de diagnóstico en los casos tempranos fue un día y en los tardíos fue de 11 días. Esta clasificación permite separar los casos por transmisión vertical (MN temprana) de aquellos atribuibles a transmisión comunitaria y/o nosocomial (MN tardía). Sin embargo, se deben considerar los periodos de incubación variables de algunos patógenos como Listeria monocytogenes (3 a 70 días) ${ }^{(15)}$.

El tiempo trascurrido entre el primer síntoma y el diagnóstico varió entre 1,5 a dos días en ambas formas clínicas, lo cual es adecuado considerando la baja letalidad y la pleocitosis moderada. Sin embargo, no podemos precisar si la punción lumbar siguió de una fuerte sospecha de meningitis en un paciente con indicadores de sepsis o por una sospecha dual de sepsis y meningitis. En ambos casos, la sospecha clínica será la regla, por lo inespecífico de los síntomas.

Se halló una predominancia del parto vaginal en $\mathrm{MN}$ temprana, considerado como una fuente importante de contaminación en mujeres portadoras de patógenos agresivos, proceso que puede originar sepsis y meningitis neonatal (9). El hallazgo de estos microorganismos en algunos casos tardíos abre un espectro de hipótesis respecto al mecanismo de colonización.

No se cuenta con una explicación para el predominio del sexo femenino, ya que, generalmente, se espera una predominancia masculina en proporción de 1,2:1 atribuido a mayor inmadurez pulmonar en neonatos varones ${ }^{(13,16)}$. Sin embargo, podría deberse al tamaño de la serie estudiada.

La tasa de letalidad fue menor a la esperada para países en desarrollo (15-30\%) y es concordante con países desarrollados $(<10 \%)^{(5,9,14)}$, siendo mayor en casos de MN temprana. Esto difiere de estudios españoles y franceses donde se encontraron tasas de letalidad de 2,3 y 2 veces mayor en MN tardía, teniendo

Tabla 3. Resultados de los exámenes en liquido cefalorraquídeo en la población de estudio con meningitis neonatal en $\mathrm{HCH}, 2008-2015$

\begin{tabular}{|c|c|c|c|}
\hline \multirow[t]{2}{*}{ Resultados } & $\begin{array}{l}\text { MN de aparición temprana } \\
(n=10)\end{array}$ & $\begin{array}{l}\text { MN de aparición tardía } \\
(n=43)\end{array}$ & \multirow[t]{2}{*}{ Valor $\mathrm{p}^{*}$} \\
\hline & Mediana (RIC) & Mediana (RIC) & \\
\hline Edad (días) & $1(1-2)$ & $11(7-19)$ & $<0,001$ \\
\hline Leucocitos $(\mu \mathrm{L})$ & $330(147-1025)$ & $170(75-472)$ & 0,15 \\
\hline PMN (\%) & $35(3-56)$ & $30(10-40)$ & 0,99 \\
\hline Glucosa (mg/dL) & $29(21-43)$ & $32(17-42)$ & 0,60 \\
\hline Proteínas (mg/dL) & $228,5(186-300)$ & $190(124-300)$ & 0,19 \\
\hline Hematíes ( $\mu \mathrm{L})$ & $47,5(12-7828)$ & $300(20-9200)$ & 0,74 \\
\hline
\end{tabular}

*Prueba $U$ de Mann Witney

MN: meningitis neonatal, PMN: polimorfonucleares, $\mathrm{HCH}$ : Hospital Cayetano Heredia, RIC: rango intercuartílico 
Tabla 4. Aislamiento del patógeno de la población de estudio con meningitis neonatal en $\mathrm{HCH}, 2008-2015$

\begin{tabular}{|c|c|c|c|c|}
\hline Patógeno aislado & Medio & $\begin{array}{l}\text { MN de aparición } \\
\text { temprana }(n=10)\end{array}$ & $\begin{array}{l}\text { MN de aparición } \\
\text { tardía }(n=43)\end{array}$ & $\begin{array}{c}\text { Total } \\
\mathrm{n} / \mathrm{N}(\%)\end{array}$ \\
\hline \multirow{2}{*}{ Listeria monocytogenes } & LCR & $2 / 10$ & $7 / 43$ & \multirow{2}{*}{$10 / 53(18,9)$} \\
\hline & Sangre & $1 / 10$ & $0 / 43$ & \\
\hline \multirow{2}{*}{$\begin{array}{l}\text { Staphylococcus coagulasa } \\
\text { negativo }\end{array}$} & LCR & $0 / 10$ & $2 / 43$ & \multirow{2}{*}{$8 / 53(15,1)$} \\
\hline & Sangre & $1 / 10$ & $5 / 43$ & \\
\hline \multirow{2}{*}{ Klebsiella sp. } & LCR & $0 / 10$ & $0 / 43$ & \multirow{2}{*}{$2 / 53(3,8)$} \\
\hline & Sangre & $0 / 10$ & $2 / 43$ & \\
\hline \multirow{2}{*}{ Streptococcus grupo B } & LCR & $1 / 10$ & $0 / 43$ & \multirow{2}{*}{$1 / 53(1,9)$} \\
\hline & Sangre & $0 / 10$ & $0 / 43$ & \\
\hline \multirow{2}{*}{ Escherichia coli } & LCR & $0 / 10$ & $0 / 43$ & \multirow{2}{*}{$1 / 53(1,9)$} \\
\hline & Sangre & $0 / 10$ & $1 / 43$ & \\
\hline \multirow{2}{*}{ Staphylococcus aureus } & LCR & $1 / 10$ & $0 / 43$ & \multirow{2}{*}{$1 / 53(1,9)$} \\
\hline & Sangre & $0 / 10$ & $0 / 43$ & \\
\hline \multirow{2}{*}{ Streptococcus viridans } & LCR & $0 / 10$ & $0 / 43$ & \multirow{2}{*}{$1 / 53(1,9)$} \\
\hline & Sangre & $1 / 10$ & $0 / 43$ & \\
\hline \multirow{2}{*}{ Streptococcus sp. } & LCR & $0 / 10$ & $0 / 43$ & \multirow{2}{*}{$1 / 53(1,9)$} \\
\hline & Sangre & $0 / 10$ & $1 / 43$ & \\
\hline \multirow{2}{*}{ Pseudomonas sp. } & LCR & $0 / 10$ & $1 / 43$ & \multirow{2}{*}{$1 / 53(1,9)$} \\
\hline & Sangre & $0 / 10$ & $0 / 43$ & \\
\hline Influenza B & IFI viral & $0 / 0$ & $1 / 6$ & $1 / 6(16,7)$ \\
\hline Parainfluenza & IFI viral & $0 / 0$ & $1 / 6$ & $1 / 6(16,7)$ \\
\hline Virus sincitial respiratorio & IFI viral & $0 / 0$ & $1 / 6$ & $1 / 6(16,7)$ \\
\hline Gram (-) & Coaglutinaciones & $0 / 2$ & $1 / 8$ & $1 / 10(10,0)$ \\
\hline
\end{tabular}

MN: meningitis neonatal, $\mathrm{HCH}$ : Hospital Cayetano Heredia, LCR: líquido cefalorraquídeo, IFI: inmunofluorescencia indirecta.

ambos mayores tamaños poblacionales ${ }^{(10,17)}$. La mayor letalidad en casos tardíos puede atribuirse a que suelen ser prematuros o de bajo peso ${ }^{(5,9,10)}$.

En nuestro estudio se halló una asociación significativa entre varios factores maternos (corioamnionitis, fiebre periparto e ITU) y el desarrollo posterior de meningitis temprana o tardía en el neonato. Nuevamente, esto concuerda con lo hallado por Polin et al., para los factores maternos infecciosos ${ }^{(5)}$. Los casos de $\mathrm{MN}$ tardía son el resultado de una infección nosocomial y/o domiciliaria, cuya frecuencia aumentará con cualquier factor que incremente la estancia hospitalaria ${ }^{(10)}$.

Una revisión refiere que más del $30 \%$ de casos pueden ser asintomáticos (17). Contrariamente, en esta serie todos fueron sintomáticos, sugiriendo mayor gravedad y sensibilidad diagnóstica. La predominancia de síntomas respiratorios altos en casos tardíos sugiere que estos hayan presentado un cuadro infeccioso respiratorio previo al desarrollo de bacteriemia y compromiso neurológico ${ }^{(5)}$.

Los estudios del LCR muestran pleocitosis moderada con hipoglucorraquia e hiperproteinorraquia, con diferencia no significativa entre las medianas de los casos tempranos y tardíos. Esta pleocitosis moderada puede deberse a una característica propia del patógeno predominante, diagnóstico oportuno o posibles casos de etiología viral no detectados. El rango de los parámetros citológicos y bioquímicos en neonatos a término y pretérmino sanos es extremadamente amplio y se sobrepone con los valores indicativos de infección ${ }^{(18)}$.

A la mayoría de pacientes se les realizó más de una punción lumbar, parte del protocolo en el $\mathrm{HCH}$, para evaluar mejoría en la carga bacteriológica. Las recomendaciones acerca de esta medida son variables, pero apoyan realizar una punción lumbar de control tras 48-72 horas de instaurada la antibioticoterapia, especialmente si no presenta mejoría clínica. En aquellos casos en los que persisten positivos los cultivos, la mortalidad es mayor ${ }^{(19)}$.

La identificación del germen fue mayor que en el 2008 en el $\mathrm{HCH}^{(1)}$ y en el rango mediano-bajo en comparación a lo reportado en otros estudios $(40-80 \%)^{(3,10)}$. Los agentes aislados difieren de lo esperado, ya que en la $\mathrm{MN}$ temprana predominan Streptococcus grupo B (GBS) y $E$. coli, en tanto que en la tardía microorganismos del medio hospitalario o domiciliario como Staphylococcus coagulasa negativo (CoNS) y Klebsiella $s p .^{(5,9,10)}$. Listeria monocytogenes es un agente habitual, reportado en $5-20 \%$ de los casos, predominando en la MN temprana (5). Sin embargo, es un agente raramente reportado en las series de países en desarrollo, posiblemente por la 
dificultad para su aislamiento ${ }^{(3)}$. Este hallazgo implica la posibilidad de portadores de listeriosis subclínica ${ }^{(20)}$.

Entre las limitaciones de este estudio podemos mencionar el tamaño limitado de la serie para una infrecuente, el subregistro del uso de corticoides prenatales, la falta de cultivos de secreciones vaginales y el subregistro de corioamnionitis confirmadas por histología. Sin embargo, los hallazgos obtenidos son de suma importancia, ya que esta es una patología de gran impacto sobre la morbi-mortalidad neonatal con alto riesgo de secuelas severas. La clínica inespecífica obliga a un alto nivel de sospecha en todo neonato con factores de riesgo para garantizar un diagnóstico oportuno y tratamiento adecuado, cubriendo los patógenos más frecuentes para así aumentar la posibilidad de supervivencia y reducir el riesgo de secuelas. A nivel nacional, se cuenta con pocas investigaciones publicadas al respecto y la predominancia de un patógeno considerado poco frecuente da cabida a ampliar las investigaciones y el seguimiento a nivel hospitalario.
La incidencia hospitalaria de MN fue 1,5 casos por mil nacidos vivos y la letalidad $3,8 \%$. Los principales síntomas fueron fiebre, hipotonía e hipoactividad. Los factores relacionados fueron fiebre materna, ITU materna y corioamnionitis. EI LCR mostró pleocitosis moderada, sin predominio polimorfonuclear, con hipoglicorraquia y proteinorraquia. Predominaron Listeria monocytogenes y Staphylococcus coagulasa negativo. Se recomienda realizar estudios prospectivos y multicéntricos para determinar incidencia, características demográficas, cambios clínicos y de LCR y factores de riesgo asociados, con el propósito de crear guías clínicas dirigidas al diagnóstico temprano y tratamiento óptimo.

Contribución de los autores: DGP ha participado en la concepción del estudio y junto con GL y MS han participado en la recolección y obtención de los resultados, análisis de los datos y redacción; MLR ha participado en la revisión final del manuscrito. Todos los autores aprobaron la versión final.

Fuentes de financiamiento: autofinanciado

Conflictos de interés: no existen conflictos de interés

\section{REFERENCIAS BIBLIOGRÁFICAS}

1. Lazo E, Guillén D, Zegarra J. Meningitis neonatal en el Hospital Nacional Cayetano Heredia. Rev Peru Pediatr. 2008;61(3):157-64.

2. Oliveros MA, Ramos R, León JL, Mazzini J, Van Oordt J, Livia C. Meningitis neonatal en la UCI del Hospital Edgardo Rebagliati Martins 1986-88. Diagnóstico. 1993;32:73-7.

3. Furyk JS, Swann O, Molyneux E. Systematic review: neonatal meningitis in the developing world. Trop Med Int Health. 2011;16(6):672-9. doi: 10.1111/j.1365-3156.2011.02750.x.

4. Ku LC, Boggess KA, Cohen-Wolkowiez. Bacterial Meningitis in infants. Clin Perinatol. 2014;42(1):29-45. doi: 10.1016/j.clp.2014.10.004

5. Polin RA, Harris MC. Neonatal bacterial meningitis. Semin Neonatol. 2001; 6:157-72.

6. Simonsen KA, Anderson AL, Delair SF, Davies HD. Early-onset neonatal sepsis. Clin Microbiol Rev. 2014;27(1):21-47. doi: 10.1128/CMR.00031-13.

7. Dong Y, Speer CP. Late-onset neonatal sepsis: recent developments. Arch Dis Child Fetal Neonatal Ed. 2015;100(3):F257-63. doi: 10.1136/ archdischild-2014-306213.

8. Bell WE, McCormick WS. Neonatal Meningitis. En: Neurologic infections in children. Philadelphia: Elsevier;1981. p. 105-33.
9. Nizet V, Klein JO. Bacterial sepsis and meningitis. En: Remington and Klein's Infectious Diseases of the Fetus and Newborn. Philadelphia: Elsevier;2016. p. 217-71.

10. Coto GD, Lopez JB, Fernández B. Meningitis neonatal. Estudio epidemiológico del Grupo de Hospitales Castrillo. An Esp Pediatr. 2002;56:55663.

11. Krebs VL, Costa GA. Clinical outcome of neonatal bacterial meningitis according to birthweight. Arq Neuropsiquiatr. 2007;65(4B):1149-53.

12. Vargas-Machuca JA, Tavera Salazar M, Carrasco Gamarra M.Mortalidad neonatal en el Perú y sus departamentos 2011-2012 [Internet]. Lima: Dirección General de Epidemiología; 2013. [citado el 10 de octubre del 2016). Disponible en: http://www.unicef.org/peru/spanish/ Mortalidad-Neonatal-en-el-Peru-y-susdepartamentos-2011-2012.pdf

13. Holt DE, Halket $S$, dxe Louvois J, Harvey D. Neonatal meningitis in England \& Wales: 10 years on. Arch Dis Child Fetal Neonatal Ed. 2001;84(2):F85-9.

14. Bentlin MR, Ferreira GL, Rugolo LM, Silva GH, Mondelli AL, Rugolo Junior A. Neonatal meningitis according to the microbiological diagnosis: A decade of experience in a tertiary center. Arq Neuropsiquiatr. 2010;68(6):882-7.
15. Tortajada C, Porta R, Riba M, Santoma MJ, Palacín E, Español M. Brote nosocomial por Listeria monocytogenes en una Unidad de Neonato. Enferm Infecc Microbiol Clin. 2012; 30(3):143-6.

16. Hong DK, Lewis DB. Developmental immunology and role of host defenses in fetal and neonatal susceptibility to infection. En: Remington and Klein's Infectious Diseases of the Fetus and Newborn. Philadelphia: Elsevier;2016. p. 81-188.

17. DulacO,LassondeM,Sarnat HB. Neonatal bacterial meningitis. Handbook of Clinical Neurology. Philadelphia: Elsevier;2013.

18. Isaacs $\mathrm{D}$. The management of neonatal meningitis. Curr Paediatr. 2000;10:96-103.

19. Greenberg RG, Benjamin DK Jr, CohenWolkowiez M, Clark RH, Cotten CM, Laughon $\mathrm{M}$, et al. Repeat lumbar punctures in infants with meningitis in the neonatal intensive care unit. J Perinatol 2011; 31(6):425-9. doi: 10.1038/jp.2010.142.

20. Kollmann TR, Mailman TL, Bortolussi R. Listeriosis. En: Remington and Klein's Infectious Diseases of the Fetus and Newborn. Philadelphia: Elsevier;2016. p. 457-74.

Correspondencia: Maggie Schweig

Dirección: Jacinto Lara 328, Dpto. 402.

Lima 27, Perú

Correo electrónico:mschweig@gmail.com 